\title{
Membranes of unification
}

\author{
Peter Nick $^{1}$
}

Received: 22 November 2016/Accepted: 22 November 2016/Published online: 29 November 2016

(C) Springer-Verlag Wien 2016

When, more than three centuries ago, Robert Hooke baptised the small chambers his microscope had uncovered in his cork specimen as cellulae, he might have imagined the small departments of a monastery hosting monadic individuals. The later finding that, even in non-walled cells, membranes separate the secluded interior from the attacks of the outer world introduced membranes as tools for delineation. Even within a cell, membrane-surrounded compartments enable the coexistence of different chemistries that otherwise would be incompatible; it is separation by membranes that allows to cleave peptide bonds in the acidic environment of a lysosome, whereas a few microns apart, ribosomes assemble amino acids into new proteins. However, separation is only one way to look onto membranes - with the same justification, they might be seen as interfaces joining different spaces in a dynamic manner as is the case for the endoplasmic reticulum (ER). This most prominent of the endomembrane systems can connect different organelles within a cell creating ever-changing interactions between compartments. In plant cells, the ER can, in addition, connect neighbouring cells as so called desmotubules spanning through the plasmodesmata. Actually, the symplastic continuity within a plant tissue can be conceived as a gigantic membranous network, organising only partially separated functional units. This way to look at plant cells has moved quite far from the original monastic concept of Hooke's cellulae. Two contributions in the current issue address the unifying and integrating power of the

Handling Editor: Peter Nick

Peter Nick

peter.nick@kit.edu

1 Institut für Botanik I, Universität Karlsruhe, Kaiserstraße 2, 76128 Karlsruhe, Germany endoplasmic reticulum - both inside individual cells and on the supracellular level.

In their review article, Griffing et al. (2016) in the current issue not only give a comprehensive overview on the different functional and organisational aspects of the plant ER but also show how methodological constraints and advances have shaped our concepts of this largest membrane organelle. While fluorescent microscopy had allowed first insights into the dynamic nature of the plant endoplasmatic reticulum (Lichtscheidl and Url 1990), the full dynamics of this organelle became clear with the possibilities of GFP technology. They dissect some of the mechanisms underlying the dynamic geometry of the ER-and consider, in addition to cytoskeleton-dependent modelling, also biophysical factors such as surface tension or shapes of minimal energy. It seems that the ER can connect with most organelles in a transient manner. In plants, this dynamic remodelling is even further accentuated by cytoplasmic streaming. The authors combine these aspects in addressing two phenomena, where ER remodelling is prominent - tip growth and biotic response, such as viral movement or symbiotic interaction with Rhizobia. Mechanistic insight and functional context of ER organisation is not only exciting and relevant; it also leads to aesthetically appealing images, one of which has been selected as front matter for the current volume, because beauty is a central motivator for cell biological research.

The role of the ER as intercellular connector is addressed by Liu et al. (2016) in the current issue. In this work, the senior author returns to a seminal paper published more than three decades ago, where the continuity of the ER with the desmotubule had been shown (Overall et al. 1982). In the current work, they look at localisation and function of calnexins, which, together with their homologue calreticulin, are major calcium-binding proteins in the ER involved in protein folding. Since calreticulin is localised to the 
desmotubules, the authors ask, whether calnexin is found in the plasmodesmata as well. Further, they use calnexin T-DNA insertion lines in Arabidopsis to address a potential function in plasmodesmatal transport by measuring GFP transport across cell layers in those mutants or in overexpression lines. Although calnexin was found in the ER membrane, and also decorated the plasmodesmata, there was no specific association with the desmotubules, but rather calnexin was globally localised in the ER membrane. Although the transport of GFP seemed to be independent of the expression level of calnexin, in the mutants, there was a modulation of callose deposition at the plasmodesmata. Thus, while calnexin did not show an obvious role in transport itself, it might exert some regulatory function for cell-to-cell conductivity, which is possibly linked with its role in calcium homeostasis. Supracellular integration might not only be regulated at the plasmodesmata themselves, but might alternatively integrate signals from the ER in a more global manner.

\section{Compliance with ethical standards}

Conflict of interest The author declares that there is no conflict of interest.

\section{References}

Griffing L, Lin CP, Perico C, White R, Sparkes I (2016) Plant ER geometry and dynamics: biophysical and cytoskeletal control during growth and biotic responsee. Protoplasma, current issue

Lichtscheidl IK, Url WG (1990) Organisation and dynamics of cortical endoplasmic reticulum in inner epidermal cells of onion bulb scales. Protoplasma 157:203-215

Liu D, Smith P, Barton D, Day D, Overall RL (2016) Characterisation of Arabidopsis Calnexin 1 and Calnexin 2 in the endoplasmic reticulum and at plasmodesmata. Protoplasma, current issue

Overall RL, Wolf J, Gunning BES (1982) Intercellular communication in Azolla roots. I. Ulstrastructure of plasmodesmata. Protoplasma 111: 134-150 